\title{
Metodologia para o balanceamento de linhas de montagem multi-modelo em ambientes de customização em massa
}

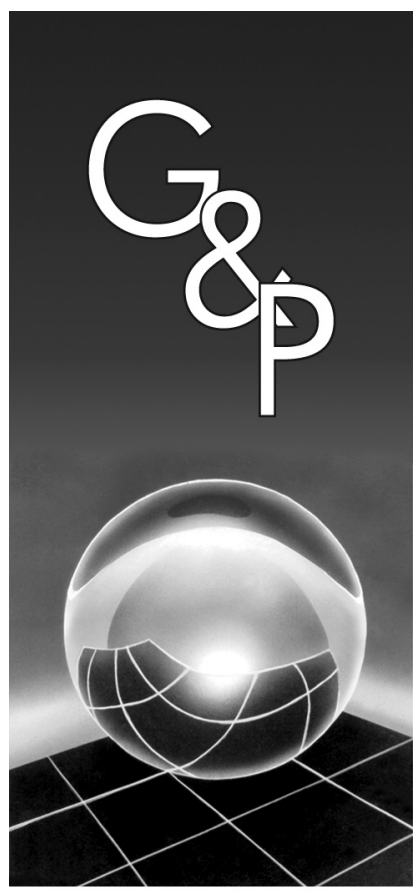

\author{
Melissa Petry Gerhardt \\ Flávio Sanson Fogliatto \\ Marcelo Nogueira Cortimiglia
}

Resumo

A implementação da customização na produção requer flexibilidade no processo produtivo. Esta pode ser adquirida através da adoção de linhas de montagem capazes de alternar diferentes modelos de um mesmo produto: as linhas de montagem multi-modelo. Neste tipo de sistema produtivo, a distribuição de tarefas entre as estações de trabalho tende a ser mais complexa, e os algoritmos de balanceamento de linhas uni-modelo tornam-se inadequados. Este artigo apresenta uma metodologia para balanceamento em linhas de montagem multi-modelo, a partir de procedimentos disponíveis na literatura e de proposições originais. Também propõe uma classificação dos mais recentes algoritmos desenvolvidos para o balanceamento de linhas de montagem multi-modelo em relação às suas principais características. A metodologia proposta tem sua aplicação ilustrada através de um estudo de caso na indústria de eletrodomésticos.

Palavras-chave: Balanceamento de linhas multi-modelo. Classificação de algoritmos. Layout. Otimização.

\section{Introdução}

Nas últimas décadas, tem-se observado entre as empresas de manufatura profundas transformações em seus ambientes econômicos e tecnológicos. Em termos econômicos, estão inseridas em um processo de globalização e unificação de mercados. Em face a esse novo ambiente, as empresas têm sido pressionadas a reavaliar suas estratégias básicas de manufatura, de forma a encontrar uma posição de mercado competitiva (FRUTOS; BORENSTEIN, 2004; JIAO et al., 2003).

Neste contexto, a customização da produção se destaca como uma estratégia fundamental para as empresas de manufatura, visto que produtos diferenciados tendem a apresentar melhor potencial de aceitação pelo mercado consumidor (DA SILVEIRA et al., 2001). O objetivo da customização é produzir itens que atendam às preferências individuais dos clientes a preços similares aos de produtos padronizados e, desta forma, obter um diferencial competitivo. Para isso, são necessários sistemas de produção flexíveis, capazes de produzir diversas versões de um mesmo produto. A configuração de linha de produção multi-modelo (MERENGO et al., 1999;
BECKER; SCHOLL, 2006) é uma opção apropriada a esta necessidade de flexibilização.

Por outro lado, o balanceamento de linhas de montagem multi-modelo, isto é, a distribuição das tarefas em estações de trabalho, de maneira que cada estação utilize aproximadamente o mesmo tempo para realizar as tarefas nela alocadas, tem se apresentado como um problema clássico nas indústrias (PASTOR et al., 2002, por exemplo). O presente trabalho tem por objetivo abordar esse problema através da proposição de uma metodologia para o balanceamento de linhas de montagem multi-modelo, a qual é ilustrada através de um estudo de caso em uma empresa do setor de eletrodomésticos.

A metodologia proposta apresenta duas contribuições relevantes que merecem destaque: a) oferece uma estrutura de decisão que orienta o usuário quanto à utilização do algoritmo de balanceamento multi-modelo mais adequado a seu cenário de aplicação; e b) propõe indicadores que permitem quantificar a melhoria resultante do balanceamento da linha multi-modelo.

$\mathrm{O}$ artigo também traz uma revisão acerca do estado da arte do balanceamento de linhas multi-modelo e propõe 
uma classificação dos algoritmos de balanceamento multi-modelo, com base no tratamento dado aos tempos de execução das tarefas que compõem o processo produtivo. Dois grandes grupos de algoritmos são propostos: aqueles que consideram tempos determinísticos de execução das tarefas e os que tratam tais tempos de forma estocástica. A classificação permite operacionalizar um dos principais passos da metodologia aqui proposta: a escolha do procedimento para o balanceamento da linha de montagem.

A estrutura do restante deste artigo é a seguinte. A seção 2 traz um referencial teórico, no qual se destaca uma proposição de classificação dos algoritmos de balanceamento de linhas multi-modelo disponíveis na literatura. A metodologia proposta é apresentada na seção 3, paralelamente ao estudo de caso, onde o balanceamento de uma linha de produção de equipamentos refrigeradores é analisado. A conclusão encerra o artigo, na seção 4.

\section{Customização em massa, linhas multi- modelo e classificação dos algoritmos de balanceamento}

Para atender a demanda por produtos customizados e de baixo custo, um novo sistema de produção foi concebido por Davis (1989), a customização em massa (CM). Segundo autores como Da Silveira et al. (2001) e Frutos e Borenstein (2004), a CM consiste em um novo conceito na indústria, o qual objetiva fornecer produtos e serviços diferenciados através de processos flexíveis, em diferentes volumes e a baixo custo.

A principal característica de um produto ou serviço elaborado em um sistema de CM é o número de opções de escolha oferecido aos clientes. O número de opções proporcionadas, assim como a forma como estas serão oferecidas aos clientes, determinarão o nível de customização a ser praticado em um produto ou serviço, bem como a conseqüente demanda por flexibilidade dentro da organização. Lampel e Mintzberg (1996) classificaram a CM dentro de cinco categorias distintas, em nível crescente de customização oferecida ao cliente: Customização Pura, Adaptada ou Padronizada e Padronização Segmentada ou Pura.

No entanto, o nível de customização oferecido ao cliente não é o único determinante do sucesso da implementação da CM em uma organização. Entre os elementos habilitadores da implementação da CM, um dos principais é a flexibilidade na manufatura. A utilização de uma configuração flexível na manufatura surge como ponto chave para produção de produtos diferenciados, e se constitui em característica essencial para responder aos pequenos ciclos de vida dos produtos, pequenos a médios volumes de produção, mudanças no padrão de demanda e à grande variedade de modelos e opções de produto.
Desta forma, o futuro econômico da indústria aponta na direção de sistemas flexíveis de produção, técnicas avançadas de manufatura e habilidade intensiva para a fabricação de produtos customizados (GERWIN, 1993). A flexibilidade é, neste contexto, um habilitador para o baixo custo de produtos customizados, maior oferta de produtos diferenciados e fabricação de produtos de tecnologia superior, direcionados a nichos de mercado.

$\mathrm{O}$ arranjo físico da manufatura, ou layout, surge como importante fator que pode tornar-se a diferença entre um sistema produtivo flexível ou não. No layout por produto, considerado a melhor configuração para a produção contínua e repetitiva de um único produto, as estações de trabalho são seqüenciadas, de modo que os produtos são montados de acordo com sua movimentação pelas estações, sendo cada uma delas responsável pela execução de uma parcela do trabalho. Este tipo de layout, associado a uma linha multi-modelo, permite a operacionalização da flexibilização de manufatura, desde que a distribuição das tarefas nas estações de trabalho seja otimizada.

Linhas multi-modelo são geralmente utilizadas para montagem de dois ou mais modelos diferentes de um mesmo produto, cujos itens de produção se alteram de modelo para modelo continuamente na linha de montagem (SARKER; PAN, 1998). A quantidade de cada modelo produzido pela linha é usualmente baixa, devido ao desejo do cliente por modelos variados e sua correspondente demanda.

Formalmente, o problema de balanceamento de uma linha de montagem multi-modelo pode ser definido da seguinte maneira: dado o número de modelos, as suas tarefas associadas, o tempo para realização de cada tarefa e suas relações de precedência, o problema consiste em alocar as tarefas a uma determinada seqüência de estações, de modo que as relações de precedência sejam satisfeitas e a capacidade otimizada (EREL; GOKCEN, 1999; BECKER; SCHOLL, 2006). A diferença básica entre um problema de balanceamento de linha de montagem uni-modelo para um multi-modelo reside no diagrama de precedências (VAN ZANTE-DE FOKKERT; DE KOK, 1997). Em uma linha de montagem multimodelo, todos os modelos possuem o seu próprio diagrama de precedências, e o balanceamento não deve violar suas respectivas restrições. Em uma linha uni-modelo, no entanto, somente um diagrama de precedências é gerado, sendo mais simples de se obter o balanceamento. A fim de resolver o problema de balanceamento de linhas multimodelo, foram desenvolvidos diversos algoritmos. Uma classificação dos mais recentes e expressivos é apresentada na sequiência, e serve como base para a metodologia proposta neste artigo.

Segundo Sprecher (2003), são considerados tradicionalmente dois tipos de problema de balanceamento em linhas de montagem. O primeiro (Tipo 1) tem como 
objetivo minimizar o número de estações de trabalho ao longo da linha de produção. Se a estação for ocupada por um trabalhador, o objetivo pode ser interpretado como minimizar os custos relativos à mão-de-obra. $\mathrm{O}$ segundo (Tipo 2) parte de um número de estações de trabalho fixo e tem como objetivo maximizar a taxa de produção ou, de forma equivalente, minimizar o tempo de ciclo.

Um dos primeiros autores a estudar o problema, Thomopoulos $(1967 ; 1970)$ desenvolveu um trabalho de significativa importância. Em seu trabalho seminal (THOMOPOULOS, 1967), o autor propõe uma adaptação da heurística de balanceamento uni-modelo desenvolvida por Kilbridge e Wester (1961) para o balanceamento de linhas multi-modelo. Já em seu segundo trabalho, Thomopoulos (1970) procura facilitar o balanceamento através de uma seqüência de passos padronizados para alocação das tarefas às estações de trabalho. Para cada estação, o algoritmo gera um número finito de possíveis combinações de tarefas, respeitando as relações de precedência e o tempo de ciclo, selecionando a combinação que gera a distribuição de trabalho mais equilibrada entre as estações. A contribuição mais importante de Thomopoulos (1970) diz respeito ao fato de que, ao se alocar tarefas às estações separadamente para cada modelo, gera-se uma quantidade de trabalho desigual ao longo da linha. A seqüência de passos padronizada é possível somente através da união dos diagramas de precedência referentes a cada modelo em um diagrama de precedências equivalente.

A partir desse desenvolvimento inicial, diversos algoritmos para o balanceamento de linhas multi-modelo foram propostos. Lehman (1969) apresentou uma formulação baseada em programação linear para o problema de balanceamento de linhas multi-modelo, com o objetivo de minimizar o custo total associado ao desbalanceamento, atraso no seqüenciamento e aprendizado das tarefas. Robert e Villa (1970) apud Ghosh e Gagnon (1989) apresentaram uma formulação baseada em programação inteira que minimiza o excesso de trabalho existente nas estações. Os autores usaram o algoritmo da rota mais curta desenvolvido por Gutjar e Nemhauser (1964) para encontrar uma solução ótima para o problema. Macaskill (1972) propôs uma heurística para a implementação computacional da alocação de tarefas a estações de trabalho.

Como forma de apresentar os mais recentes algoritmos desenvolvidos para o balanceamento de linhas de montagem, propõe-se uma classificação em que os algoritmos são inicialmente tipificados de acordo com os tempos de realização das tarefas, determinísticos ou estocásticos. O último passo da classificação é feito segundo a circunstância do problema, que pode ser simples ou genérica. Os casos genéricos podem incluir fatores como paralelismo de estações de trabalho, restrições de zone- amento e formato da linha. O esquema de classificação proposto para linhas de montagem multi-modelo, bem como seus respectivos algoritmos, é apresentado na Figura 1.

O problema de balanceamento em forma determinística pode ser definido da seguinte maneira: dado um determinado número de tarefas, cada uma com seu respectivo tempo fixo de realização e um diagrama com as relações de precedência entre elas, o problema consiste em alocar as tarefas a uma determinada sequiência de estações de trabalho, de forma que as relações de precedência sejam satisfeitas e a capacidade da linha otimizada (EREL; SARIN, 1998).

Já o problema de balanceamento de linhas de montagem na sua forma estocástica pode ser definido da seguinte maneira: dado um número determinado de tarefas com tempos de realização descritos por distribuições de probabilidade e um diagrama de relações de precedência, o problema consiste em alocar as tarefas a uma determinada sequiência de estações de trabalho, de forma que as relações de precedência sejam obedecidas e a capacidade da linha otimizada (EREL; SARIN, 1998). Esta versão é mais realista em linhas de montagem manuais, em que o tempo de execução das tarefas costuma não ser constante. Com a introdução de tempos estocásticos, outros pontos se tornam relevantes ao problema, como: o trabalhador exceder o tempo de ciclo determinado, a instalação de estoques intermediários e o comprimento das estações de trabalho.

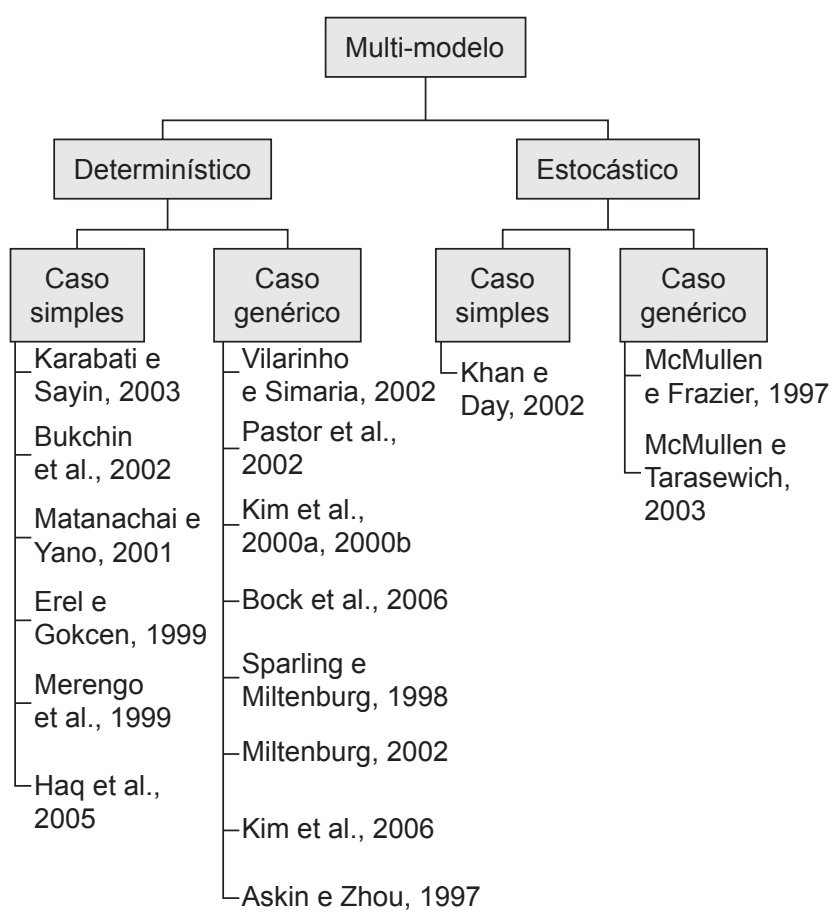

Figura 1. Classificação dos algoritmos de balanceamento de linhas multi-modelo. 


\subsection{Tempo determinístico - caso simples}

A solução para o problema de balanceamento de linhas multi-modelo apresentada por Karabati e Sayin (2003) se aplica especificamente a ambientes em que o transporte de materiais pela linha é feito de forma sincronizada, isto é, nos quais o dispositivo transportador movimentase periodicamente por um tempo pré-determinado. Em conjunto com o problema de balanceamento, os autores consideram a seqüência na qual os diferentes modelos são introduzidos na linha, integrando a alocação das tarefas às estações de trabalho e às decisões de sequienciamento dos modelos. A formulação matemática desenvolvida pelos autores tem como objetivo minimizar o tempo total de ciclo, dada uma seqüência de modelos inicial, sendo operacionalizada pela combinação dos vários modelos em um único, através da soma dos tempos de operação.

O problema de balanceamento de linhas de montagem multi-modelo em um ambiente make-to-order foi introduzido por Bukchin et al. (2002). Os autores admitem que, neste ambiente, os trabalhadores devem ser mais versáteis que os trabalhadores das linhas tradicionais, o que possibilita alocar o mesmo elemento de trabalho a diferentes estações. Considerando este fato, as tarefas de montagem são divididas em dois conjuntos: o primeiro contém as tarefas que devem ser alocadas a uma estação específica, independente do modelo do qual ela necessita; o segundo é formado pelas tarefas que podem ser alocadas a qualquer estação. Conseqüentemente, a heurística desenvolvida pelos autores com o objetivo de minimizar o número de estações de trabalho ao longo da linha tem o seu procedimento desenvolvido em três estágios. No primeiro, a partir da união dos diagramas de precedência dos diferentes modelos em um diagrama equivalente, é alocado o primeiro conjunto de tarefas às estações. Após, o balanceamento de cada modelo é feito separadamente, designando as tarefas do segundo conjunto às estações, considerando as tarefas já alocadas. No último estágio, a solução é melhorada através de um estudo dos vizinhos, realizando mudanças na alocação das tarefas do primeiro conjunto.

O algoritmo utilizado por Matanachai e Yano (2001) tem como objetivos: a) tornar razoável o balanceamento da carga de trabalho entre as estações; e b) facilitar a determinação de sequiências diárias de modelos que forneçam uma carga de trabalho estável na linha de montagem. A solução é obtida através da construção de diferentes conjuntos de tarefas para cada estação de trabalho; a estação que apresentar o conjunto de tarefas que gere o melhor valor na função objetivo será utilizada como base para gerar os conjuntos das estações subseqüentes. Depois que a solução inicial é encontrada, implementa-se um processo para melhoria do resultado através da transferência de tarefas de estação à estação, a fim de melhorar o valor da função objetivo. Os autores consideram esta- ções fechadas, um sistema de transporte sincronizado e uma combinação dos diagramas de procedências dos diferentes modelos.

Erel e Gokcen (1999) utilizam a determinação da rota mais curta a fim de encontrar a solução ótima para o problema de balanceamento de linhas de montagem multimodelo. O procedimento pressupõe que existem tarefas comuns entre os modelos; no entanto, como os tempos de realização das tarefas podem variar de acordo com o modelo, elas devem ser alocadas na mesma estação de trabalho. O problema de linhas multi-modelo é transformado em um problema de linha com um único produto, a partir da união dos diagramas de precedência. A formulação é baseada no algoritmo de Gutjahr e Nemhauser (1964), desenvolvido para balanceamentos de linhas de modelo único.

A metodologia proposta por Merengo et al. (1999) pode ser aplicada a linhas de montagem que utilizem como sistema de transporte de materiais uma das três alternativas: a) transporte contínuo, em que a velocidade da linha é constante; b) transporte sincronizado, em que o dispositivo transportador movimenta-se periodicamente; e c) transporte não sincronizado, no qual o dispositivo transportador se move de acordo com a velocidade do trabalhador para realizar determinada tarefa. $\mathrm{O}$ algoritmo apresentado pelos autores possui os seguintes objetivos: minimizar a taxa de trabalhos incompletos (linhas com transporte contínuo ou sincronizado), minimizar a probabilidade de eventos de obstrução da linha (linhas com transporte não sincronizado), e diminuir o número de estações ao longo da linha. A operacionalização do algoritmo exige a união dos diagramas de precedência dos diferentes modelos em um diagrama equivalente.

Finalmente, Haq et al. (2005) propõem uma abordagem mista, em que uma solução de balanceamento derivada de um método heurístico com combinação de diagramas de precedência é introduzida aleatoriamente na parametrização de um algoritmo genético. Através de um método computacional de busca que simula o funcionamento da evolução biológica natural, foi possível resolver um problema de balanceamento de linha de montagem multimodelo do Tipo 1 sem restrições ou objetivos adicionais.

\subsection{Tempo determinístico - caso genérico}

Vilarinho e Simaria (2002) apresentam um modelo de programação matemática para o problema de balanceamento de linhas de montagem multi-modelo, o qual admite estações de trabalho paralelas e restrições de zoneamento. $\mathrm{O}$ modelo permite que se defina a priori o número máximo de réplicas das estações de trabalho, além das condições para que as réplicas ocorram. $\mathrm{O}$ algoritmo apresenta dois objetivos específicos: a) minimizar o número de estações de trabalho ao longo da linha; e b) dividir a carga de trabalho entre as estações da melhor 
forma possível. Devido a natureza combinatória do problema, um procedimento em dois passos é desenvolvido, sendo cada passo dedicado ao atingimento de cada objetivo. O modelo é operacionalizado pela união dos diagramas de precedência dos diferentes modelos.

Pastor et al. (2002) fazem uso de uma estratégia metaheurística, com uma abordagem de busca tabu otimizando o resultado de procedimentos heurísticos empregados. Os autores tratam do problema de balanceamento de linhas multi-modelo com objetivos adicionais de uniformização da carga de trabalho e otimização da dispersão das tarefas na produção dos diferentes modelos, além de restrições de disponibilidade de espaço e ferramentas nas estações, e existência de estoques intermediários.

O algoritmo apresentado por Kim et al. (2000a, 2000b) procura unir o balanceamento ao seqüenciamento dos modelos a serem lançados na linha. A metodologia é aplicada a linhas de montagem que possuam o formato em "U". O algoritmo utilizado pelos autores, denominado evolucionário simbiótico, simula o processo evolutivo da natureza em que as espécies possuem influência umas sobre as outras na interação e adaptação com o meioambiente. Os autores consideram que este processo é similar a situações nas quais diversos problemas estão relacionados entre si, de tal forma que uma pequena mudança na solução de um problema isolado possa vir afetar a solução dos outros problemas envolvidos.

Bock et al. (2006) também abordam o problema de balanceamento juntamente com a seqüência de introdução dos modelos na linha, levando em consideração possíveis distúrbios como: falta de operadores, interrupções não programadas e falta de material durante o processo de produção. Os autores consideram a possibilidade de emprego de operadores flutuantes, os quais podem ser temporariamente alocados a uma estação a fim de aumentar sua capacidade. $\mathrm{O}$ modelo sugerido objetiva minimizar os custos de produção através de abordagens heurísticas próprias para uso em tempo real e em ambientes dinâmicos, nos quais os recursos computacionais devem ser utilizados da maneira mais eficiente possível. O procedimento iterativo de otimização da solução faz uso de uma busca de variáveis de vizinhança empregando computação paralela.

O problema de balanceamento de linhas multi-modelo que possuem o formato em "U” é abordado por Sparling e Miltenburg (1998). O objetivo proposto consiste em alocar as tarefas necessárias à produção de todos os modelos a um número mínimo de estações de trabalho. Para isto, os autores apresentam um algoritmo de quatro passos, baseado na proposta de Thomopoulos (1970) para o problema de balanceamento de linhas de montagem multi-modelo. Os primeiros dois passos transformam o problema multi-modelo em um problema uni-modelo equivalente. $\mathrm{O}$ terceiro passo encontra a solução ótima para este problema utilizando um algoritmo de branch and bound. $\mathrm{O}$ quarto passo ajusta o balanceamento obtido para o problema original multi-modelo. Mais recentemente, Miltenburg (2002) voltou a abordar o problema de balanceamento de linhas multi-modelo em formato "U', juntamente com o problema de seqüenciamento de modelos, em uma abordagem até então inédita na literatura. $\mathrm{O}$ autor faz uso de algoritmos genéticos, uma vez que o número total de soluções para uma instância prática do problema é demasiadamente grande. Uma abordagem similar para balanceamento e seqüenciamento de linhas multi-modelo em formato "U" é apresentada por Kim et al. (2006). Os autores empregam uma evolução do modelo de algoritmo genético evolucionário simbiótico, o algoritmo evolucionário endosimbiótico, com resultados superiores àqueles usualmente associados às abordagens tradicionais hierárquicas e às abordagens com algoritmos genéticos simbióticos.

Askin e Zhou (1997) propõem o uso de programação inteira não linear para modelar o problema de balanceamento de linhas multi-modelo quando estações de trabalho paralelas são admitidas. A função objetivo desenvolvida busca eliminar o tempo ocioso nas estações de trabalho, a partir da duplicação dos equipamentos necessários à execução das tarefas. Observa-se que a decisão de duplicar uma estação é feita não somente baseada no tempo de execução das tarefas alocadas à estação, mas também comparando o custo relativo à compra dos equipamentos necessários, com o custo de se deixar uma estação ociosa durante alguns períodos de tempo. Uma heurística é desenvolvida para criar as estações paralelas e alocar as tarefas, sendo operacionalizada pela união dos diagramas de precedência.

\subsection{Tempo estocástico - caso simples}

Khan e Day (2002) introduzem uma metodologia baseada no conhecimento obtido, denominada Knowledge Based Design Methodology (KBDM), para o balanceamento de linhas. A metodologia pode ser utilizada tanto para linhas manuais quanto para linhas automatizadas. $\mathrm{O}$ primeiro passo do algoritmo consiste na seleção do sistema de montagem a ser utilizado, para a partir disso, determinar tempos de ciclo e a necessidade ou não de estações paralelas. O objetivo é determinar um número econômico de estações de trabalho, além de um design detalhado da linha de montagem. A metodologia é exclusivamente baseada em uma avaliação inicial, a qual fornece os dados necessários para o design apropriado da linha de montagem.

\subsection{Tempo estocástico - caso genérico}

O balanceamento de linhas multi-modelo com tempos de duração estocásticos das tarefas e com a possibilidade de utilizar estações de trabalho paralelas foi introduzido 
por McMullen e Frazier (1997). A técnica apresentada pelos autores é uma adaptação da heurística desenvolvida por Gaither (1996), a qual utilizava tempos determinísticos e linhas uni-modelo. O objetivo dos autores é minimizar o número de estações de trabalho, dado um determinado tempo de ciclo para linhas com altas taxas de produção. A operacionalização do algoritmo consiste em primeiramente reduzir o problema multi-modelo a uni-modelo; então são examinados os efeitos de se alocar tarefas a cada estação, de acordo com regras de seleção de tarefas pré-estabelecidas.

Por fim, o algoritmo desenvolvido por McMullen e Tarasewich (2003) utiliza a técnica Ant Colony Optimization (ACO), ou seja, Otimização da Colônia de Formigas, a fim de tratar o problema de balanceamento de linhas de montagem com fatores como: estações de trabalho paralelas, atividades com duração estocástica e modelos mistos. A metodologia é inspirada no comportamento social dos insetos como forma de alocar tarefas às estações de trabalho, de forma a otimizar o desempenho da linha.

\section{Metodologia proposta e aplicação prática}

De modo geral, algoritmos de balanceamento de linhas multi-modelo variam consideravelmente quanto aos dados de entrada necessários e aos procedimentos para obtenção da solução final. Cada procedimento é desenvolvido de acordo com características específicas da linha e com o objetivo que se pretende atingir. A aplicabilidade do balanceamento de linhas multi-modelo pressupõe o atendimento de três condições principais: a) fabricação de um produto com número fixo e conhecido de modelos; b) alternância de modelos do produto em fabricação; e c) baixa rotatividade entre os funcionários que operam a linha.

A partir destas condições, desenvolveu-se a metodologia para balanceamento de linhas de produção multi-modelo proposta neste trabalho, a qual pode ser visualizada no fluxograma apresentado na Figura 2. Os detalhes de cada passo operacional são indicados na sequiência. Em paralelo ao detalhamento da metodologia, apresenta-se sua aplicação em um estudo de caso. Optou-se por esta estratégia de apresentação para evitar

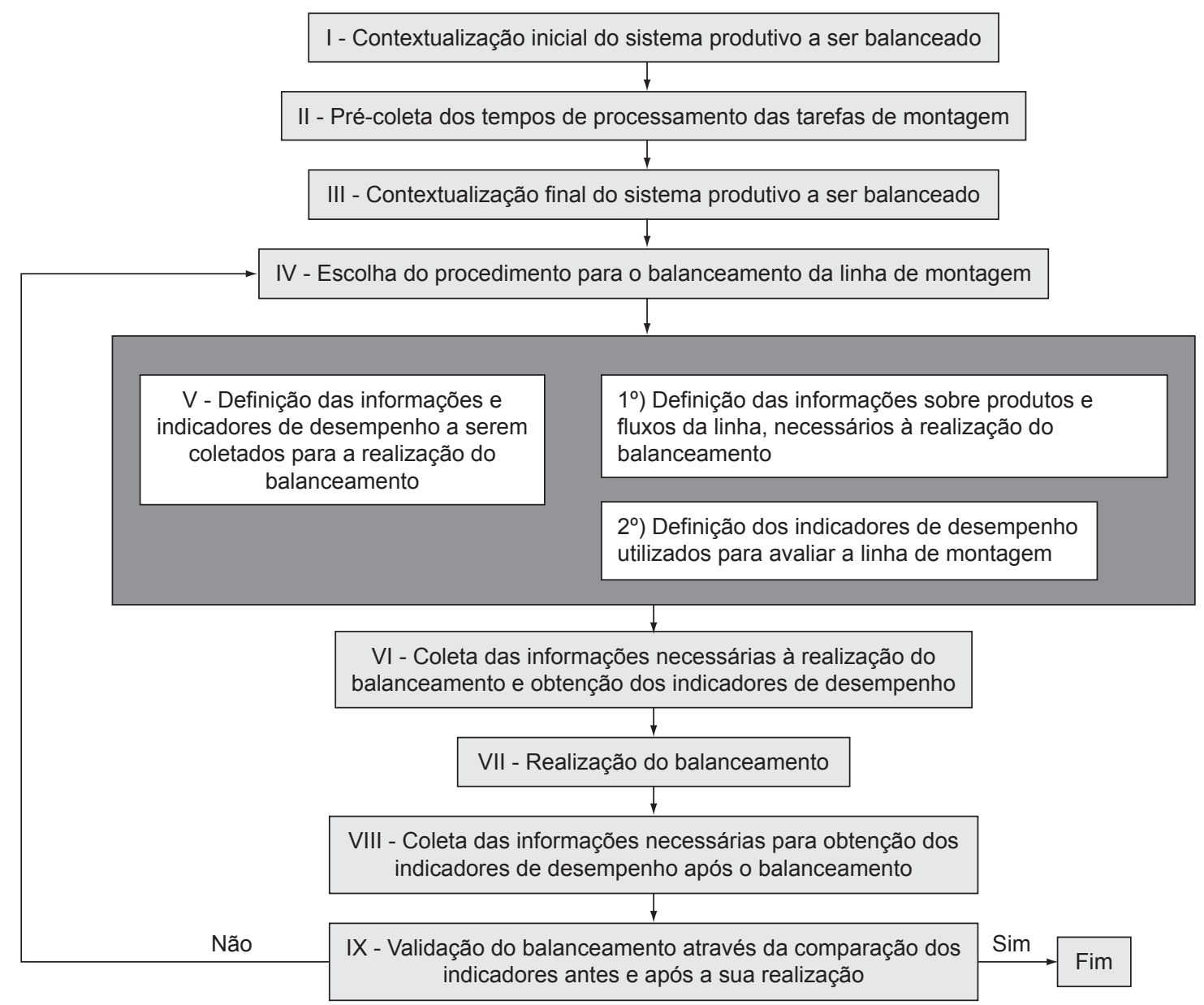

Figura 2. Fluxograma da metodologia proposta. 
redundâncias e objetivar a demonstração da metodologia, já que algumas de suas etapas são auto-explicativas e, assim, plenamente visualizáveis através do exemplo.

$\mathrm{O}$ estudo de caso foi realizado em uma empresa de eletrodomésticos localizada no sul do país, cuja atividade principal consiste na fabricação de equipamentos condicionadores de ar. Entre os produtos oferecidos pela empresa, destacam-se aparelhos de janela, splits, self contained, chiller, fan coil, refrigeradores e freezers, e expositores. $\mathrm{O}$ estudo foi realizado pelos autores no ano de 2004 e concluído em aproximadamente quatro meses.

A primeira etapa da metodologia, contextualização inicial do sistema produtivo a ser balanceado, teve início com a definição do sistema produtivo cujo balanceamento seria realizado. Em consulta à empresa, identificou-se a linha de produção LMTG G Visa Cooler, responsável pela fabricação de diferentes modelos de refrigeradores, como adequada para o estudo. A escolha justificou-se pelo crescimento na demanda de refrigeradores junto à empresa, o que aumentou sua relevância em termos de faturamento, tornando necessária a aplicação de ferramentas que otimizassem a capacidade produtiva da linha. Além disso, a linha de montagem em questão viabiliza tecnicamente a realização do balanceamento, pois produz mais do que um modelo do produto e possui sistema de transporte não sincronizado.

Procedeu-se, em seguida, com a pré-coleta dos tempos de processamento das tarefas de montagem, a fim de identificar a natureza dos tempos em relação à sua variabilidade. Foram identificadas as tarefas realizadas pela linha, independente do modelo produzido, a partir de entrevistas com o coordenador e operadores da linha de montagem. Listou-se um total de 40 tarefas envolvidas na fabricação dos modelos produzidos pela linha analisada. Após a identificação das tarefas de montagem, foi possível realizar a pré-coleta dos tempos de processamento.

Constatou-se, que algumas tarefas possuíam tempos de execução distintos de acordo com o modelo produzido. Nestes casos, houve a necessidade de coletar tempos de processamento para cada modelo, separadamente. A Tabela 1 apresenta uma visão parcial dos resultados. São apresentados: a numeração das tarefas, seus tempos médios de processamento, o desvio-padrão e o coeficiente de variação, calculados a partir de amostras de tamanho 10. Na Tabela 1, as tarefas identificadas com letras, após a numeração, referem-se aos casos em que verificou-se a existência de diferenças nos tempos de processamento da tarefa, de modelo para modelo, acarretando a necessidade de se coletar separadamente os tempos de processamento da tarefa.

A partir dos valores de coeficiente de variação dos tempos de processamento, determinou-se que a linha
Tabela 1. Estatísticas derivadas da pré-coleta de tempos de realização das tarefas.

\begin{tabular}{ccrc}
\hline Tarefas de montagem & $\mathbf{t}_{\text {med }}(\mathbf{s})$ & $\sigma(\mathbf{s})$ & $\mathbf{C V}(\boldsymbol{\%})$ \\
\hline 1 & 30,8 & 2,49 & 8,1 \\
$2 \mathrm{a}$ & 55,4 & 4,17 & 7,5 \\
$2 \mathrm{~b}$ & 54,3 & 2,71 & 5,0 \\
3 & 171,1 & 12,99 & 7,6 \\
4 & 61 & 3,94 & 6,5 \\
5 & 164,8 & 13,31 & 8,1 \\
6 & 135,3 & 10,65 & 7,9 \\
$7 \mathrm{a}$ & 58,1 & 4,68 & 8,1 \\
$7 \mathrm{~b}$ & 63,4 & 1,84 & 2,9 \\
$8 \mathrm{a}$ & 336 & 23,32 & 6,9 \\
$8 \mathrm{~b}$ & 32,5 & 14,29 & 4,4 \\
9 & 10,6 & 8,85 & 8,2 \\
\hline
\end{tabular}

analisada possui tempos determinísticos para as tarefas de montagem, ou seja, a variação existente entre tempos de execução das tarefas é relativamente pequena. Isso se deve ao fato de que em nenhuma das 40 tarefas o coeficiente de variação foi superior a $10 \%$, valor assumido na pesquisa como limite para a variabilidade apresentada por uma dada variável aleatória ser considerada moderada.

Para a contextualização final do sistema produtivo a ser balanceado, realizaram-se verificações de campo na empresa, as quais permitiram identificar a configuração da linha analisada como uma configuração em formato tradicional. Assim, foi possível encontrar no sistema produtivo analisado as condições para a aplicação da metodologia.

A escolha do procedimento de balanceamento da linha de montagem foi realizada da seguinte maneira: em uma primeira etapa, associou-se a linha analisada às classificações Tempo determinístico - Caso simples e Tempo determinístico - Caso genérico, listadas na Figura 1. Os critérios utilizados nas classificações incluem tanto a natureza aproximadamente determinística, quanto a configuração de linha em formato tradicional.

Após a identificação inicial dos procedimentos, foi escolhido o procedimento desenvolvido por Vilarinho e Simaria (2002) como o mais representativo da situação analisada. A escolha do procedimento se deu pelos seus objetivos, os quais consistem em balancear a linha tanto de forma vertical quanto horizontal, além de possibilitar fatores como: restrições de zoneamento e criação de estações de trabalho paralelas, caso seja necessário.

A seguir, procedeu-se com a definição das informações sobre produtos e fluxos da linha necessárias à realização do balanceamento, segundo o algoritmo de Vilarinho e Simaria (2002). São elas: a) identificação dos modelos; b) identificação das tarefas de montagem; c) tempo de processamento das tarefas de montagem; d) diagrama de 
precedência equivalente; e v) demanda diária por cada modelo.

$\mathrm{Na}$ mesma etapa, também se procedeu com a definição dos indicadores de desempenho utilizados para avaliar a linha de montagem, partindo da identificação de quais indicadores refletiriam as melhorias necessárias na linha em estudo. Neste contexto, o aumento da produtividade foi considerado de suma importância, devido ao crescimento expressivo na demanda do produto; assim, o indicador produtividade foi escolhido. Em conjunto, optou-se pelos indicadores desbalanceamento e índice de suavidade, os quais possibilitam verificar se a execução do balanceamento foi bem sucedida em relação à distribuição das tarefas às estações de trabalho.

$\mathrm{Na}$ etapa de coleta das informações necessárias à realização do balanceamento e obtenção dos indicadores de desempenho, os seguintes itens foram identificados como necessários: a) tempo de produção diário; b) tempo de ciclo; c) tempo de processamento das tarefas de montagem; e d) identificação das estações de trabalho. Em seguida, procedeu-se com a formulação da lista de informações a serem coletadas na etapa seguinte: a) identificação dos modelos; b) identificação das tarefas de montagem; c) identificação das estações de trabalho; d) tempo de processamento das tarefas de montagem; e) diagrama de precedência equivalente; f) demanda diária por cada modelo; e g) tempo de ciclo. A informação referente ao tempo de produção diário é obtida para o cálculo do tempo de ciclo.

A identificação do número de modelos produzidos pela linha de montagem foi obtida através de entrevistas com o seu coordenador, as quais possibilitaram a identificação de 4 diferentes modelos de refrigeradores fabricados pela linha; são eles: a) Modelo V400C - 1; b) Modelo V400C - 2; c) Modelo V4000; e d) Modelo C400.

A identificação das tarefas de montagem ocorreu através de entrevistas, nas quais foi possível também identificar as estações de trabalho existentes na linha de montagem. O tempo de processamento das tarefas de montagem consiste na mesma informação obtida anteriormente através da pré-coleta (Tabela 1). Devido à baixa variabilidade na tomada inicial de tempos, não foi necessário ampliar a amostragem além das 10 observações iniciais.

A demanda diária por cada modelo é a seguinte: 8 unidades do Modelo V400C-1; 22 unidades do Modelo V400C-2; 5 unidades do Modelo V400; e 20 unidades do Modelo C400. De acordo com o procedimento já selecionado, a coleta desta informação deu-se em número de unidades de cada modelo. É importante destacar que os dados refletem a demanda que se deseja atingir a partir do balanceamento da linha.

Por fim, para o cálculo do tempo de ciclo da linha foram obtidas informações a respeito do tempo disponível para a produção e da produção total por turno de trabalho. $\mathrm{O}$ tempo disponível para produção, bem como o número médio de refrigeradores produzidos por turno pela linha, atualmente, foram definidos pelo coordenador da linha como 480 minutos/turno e 42 refrigeradores (incluindo todos os modelos), resultando em 685,7 segundos de tempo de ciclo.

O diagrama de precedência equivalente para a linha de montagem analisada vem apresentado na Figura 3. O diagrama foi obtido através da aplicação do método do diagrama de precedência equivalente (THOMOPOULOS, 1970).

Em conjunto com o diagrama equivalente, foram calculados os tempos de processamento equivalentes das tarefas, obtidos de acordo com o procedimento de Vilarinho e Simaria (2002). Desse modo, primeiramente foi calculada a proporção relativa à demanda de cada modelo $\left(q_{\mathrm{m}}\right): 15 \%$ para o modelo V400C-1; $40 \%$ para o modelo V400C-2; $9 \%$ para o modelo V400; e 36\% para o modelo C400. Em seguida, realizou-se o somatório dos tempos de execução da tarefa para cada modelo, multiplicado pela respectiva proporção de demanda do modelo. Os resultados vêm apresentados na Tabela 2 .

A próxima etapa contemplou a realização do balanceamento, de acordo com o algoritmo de Vilarinho e Simaria (2002). A solução apresentada pelos autores inclui a realização de dois passos, com o objetivo de encontrar o resultado ótimo de balanceamento. Seguindo o procedimento sugerido, primeiramente aplicou-se a heurística Rank Positional Weight (RPW) aos dados coletados, com as alterações propostas para o ambiente multi-modelo.

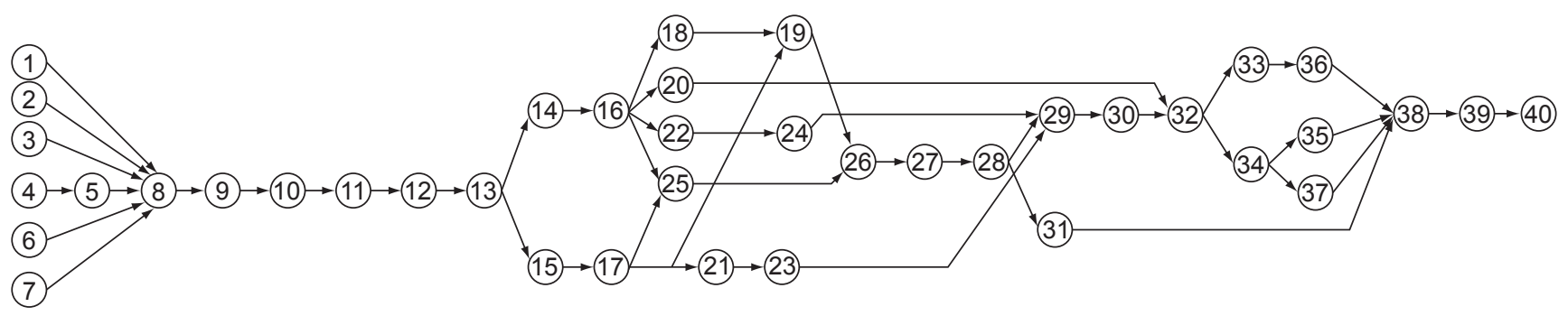

Figura 3. Diagrama de precedência equivalente. 
A partir desta heurística, obteve-se a Solução Inicial Primeiro Passo para a linha de montagem analisada. É importante destacar nesta fase que a empresa, por motivos de custo, descartou a possibilidade de duplicação de estações de trabalho.

De posse da solução inicial, foram realizados Movimentos de Troca e Transferência, de forma a atingir o

Tabela 2. Cálculo do tempo de processamento equivalente (em segundos).

\begin{tabular}{|c|c|c|c|c|c|}
\hline Tarefa & $t_{\mathrm{v} 400 \mathrm{C}-1}$ & $t_{\mathrm{v} 400 \mathrm{C}-2}$ & $t_{\mathrm{v} 400}$ & $t_{\mathrm{C} 400}$ & $\begin{array}{c}\text { Tempo de } \\
\text { processamento } \\
\text { equivalente }\end{array}$ \\
\hline 1 & 30,8 & 30,8 & 30,8 & 30,8 & 30,8 \\
\hline 2 & 55,4 & 55,4 & 54,3 & 54,3 & 54,905 \\
\hline 3 & 171,1 & 171,1 & 0 & 0 & 94,105 \\
\hline 4 & 61 & 61 & 61 & 61 & 61 \\
\hline 5 & 164,8 & 164,8 & 164,8 & 164,8 & 164,8 \\
\hline 6 & 135,3 & 135,3 & 135,3 & 135,3 & 135,3 \\
\hline 7 & 58,1 & 58,1 & 63,4 & 63,4 & 60,485 \\
\hline 8 & 336 & 336 & 321,5 & 321,5 & 329,48 \\
\hline 9 & 107,6 & 107,6 & 107,6 & 107,6 & 107,6 \\
\hline 10 & 120 & 120 & 120 & 120 & 120 \\
\hline 11 & 30 & 30 & 30 & 30 & 30 \\
\hline 12 & 225 & 225 & 225 & 225 & 225 \\
\hline 13 & 26,4 & 26,4 & 26,4 & 26,4 & 26,4 \\
\hline 14 & 0 & 0 & 522,4 & 522,4 & 235,08 \\
\hline 15 & 333,1 & 333,1 & 0 & 0 & 183,21 \\
\hline 16 & 0 & 0 & 24 & 24 & 10,8 \\
\hline 17 & 24 & 24 & 0 & 0 & 13,2 \\
\hline 18 & 0 & 0 & 105,7 & 105,7 & 47,565 \\
\hline 19 & 320,6 & 320,6 & 320,6 & 320,6 & 320,6 \\
\hline 20 & 0 & 0 & 57,6 & 57,6 & 25,92 \\
\hline 21 & 69,7 & 69,7 & 0 & 0 & 38,335 \\
\hline 22 & 0 & 0 & 71,5 & 71,5 & 32,175 \\
\hline 23 & 95,3 & 95,3 & 0 & 0 & 52,415 \\
\hline 24 & 0 & 0 & 168,2 & 168,2 & 75,69 \\
\hline 25 & 62,2 & 62,2 & 62,2 & 27,8 & 49,816 \\
\hline 26 & 230 & 230 & 230 & 230 & 230 \\
\hline 27 & 57,6 & 57,6 & 57,6 & 57,6 & 57,6 \\
\hline 28 & 186,7 & 186,7 & 186,7 & 186,7 & 186,7 \\
\hline 29 & 72,3 & 72,3 & 86,9 & 86,9 & 78,87 \\
\hline 30 & 44 & 44 & 44 & 44 & 44 \\
\hline 31 & 39,5 & 39,5 & 39,5 & 39,5 & 39,5 \\
\hline 32 & 225 & 225 & 295 & 295 & 256,5 \\
\hline 33 & 60 & 60 & 50 & 50 & 55,5 \\
\hline 34 & 108,3 & 108,3 & 96,1 & 96,1 & 102,81 \\
\hline 35 & 99,9 & 99,9 & 81 & 81 & 91,395 \\
\hline 36 & 84,4 & 84,4 & 75,5 & 75,5 & 80,395 \\
\hline 37 & 31,7 & 31,7 & 0 & 0 & 17,435 \\
\hline 38 & 89,5 & 89,5 & 47,9 & 47,9 & 70,78 \\
\hline 39 & 30,2 & 30,2 & 30,2 & 30,2 & 30,2 \\
\hline 40 & 147,5 & 147,5 & 147,5 & 147,5 & 147,5 \\
\hline
\end{tabular}

objetivo principal do problema, isto é, reduzir o número de estações de trabalho. Ao final deste estágio, obtevese a Solução Final - Primeiro Passo, com um total de 9 estações de trabalho. A Tabela 3 apresenta a alocação das tarefas às estações de trabalho, bem como os valores encontrados para o BDT para o índice de balanceamento da carga de trabalho entre as estações, $B_{\mathrm{b}-\mathrm{s}}$, e dentro de cada estação, $B_{\mathrm{w}-\mathrm{s}}$. Neste passo, o critério utilizado para avaliar as soluções vizinhas corresponde ao valor encontrado para o BDT, o qual é equivalente ao expresso no primeiro termo da função objetivo do modelo de programação matemática, apresentada por Vilarinho e Simaria (2002); os dois índices seguintes correspondem ao segundo e terceiro termos da mesma função.

No segundo passo do procedimento, novos Movimentos de Troca e Transferência foram realizados, mas com o objetivo de balancear simultaneamente a carga de trabalho entre as estações e no interior delas. A solução inicial neste passo corresponde à solução final encontrada no passo anterior. O critério utilizado para avaliar as soluções vizinhas geradas neste estágio provém diretamente dos valores obtidos para $B_{\mathrm{b}-\mathrm{s}}$ e $B_{\mathrm{w}-\mathrm{s}}$.

A Tabela 4 apresenta a solução final encontrada para a linha de montagem analisada, no segundo passo do procedimento.

A etapa seguinte envolve a coleta das informações necessárias para obtenção dos indicadores de desempenho após o balanceamento. Foi necessário realizar uma nova coleta de dados, a fim de se obter valores para os indicadores de desempenho que refletissem a nova condição da linha. As informações coletadas foram as mesmas levantadas anteriormente, ou seja: a) tempo de produção diário; b) tempo de ciclo; c) tempo de proces-

Tabela 3. Soluções inicial e final do primeiro passo.

\begin{tabular}{|c|c|c|}
\hline \multirow[t]{2}{*}{$\begin{array}{c}\text { Estações de } \\
\text { trabalho }\end{array}$} & $\begin{array}{c}\text { Solução inicial - } \\
\text { primeiro passo }\end{array}$ & $\begin{array}{l}\text { Solução final - } \\
\text { primeiro passo }\end{array}$ \\
\hline & Tarefas & Tarefas \\
\hline 1 & $4,3,5$ & $4,3,5,7,2$ \\
\hline 2 & $6,7,2,1$ & $1,6,8$ \\
\hline 3 & 8,9 & $9,10,11,12,13$ \\
\hline 4 & $10,11,12,13$ & $14,15,17,21,23$ \\
\hline 5 & 14 & $16,18,19,25,22$ \\
\hline 6 & $15,16,17,18$ & $26,27,28$ \\
\hline 7 & 19,25 & $24,29,20,30,31$ \\
\hline 8 & $26,27,22$ & $32,33,34,35$ \\
\hline 9 & $28,24,21,23$ & $36,37,38,39,40$ \\
\hline 10 & $29,20,30,32$ & - \\
\hline 11 & $34,33,35,36,31,37,38$ & - \\
\hline 12 & 39,40 & - \\
\hline $\mathrm{BDT}(\min )$ & 37,6 & 11,03 \\
\hline $\mathrm{B}_{\mathrm{b}-\mathrm{s}}$ & 0,04 & 0,14 \\
\hline $\mathrm{B}_{\mathrm{W}-\mathrm{S}}$ & 0,28 & 0,27 \\
\hline
\end{tabular}


samento das tarefas de montagem; e iv) identificação das estações de trabalho.

O tempo de produção diário e o tempo de processamento das tarefas não sofreram alterações. Para o cálculo do tempo de ciclo da linha, alterou-se a informação relacionada à produção total por turno de trabalho. A partir da realização do balanceamento, o número de refrigeradores produzidos por turno de trabalho passou a 55 unidades, incluindo todos os modelos (inicialmente, produziam-se 42 unidades). A partir dos dados, obteve-se um tempo de ciclo igual a 523,6 segundos (inicialmente, este tempo era de 685,7 segundos).

Finalmente, procedeu-se com a validação do balanceamento através da comparação dos indicadores coletados antes e após a sua realização. É importante destacar, que para o cálculo dos indicadores foram considerados os tempos de processamento equivalentes das tarefas de montagem. A Tabela 5 apresenta os valores encontrados para cada indicador antes e após a realização do balanceamento, bem como a melhoria obtida nos mesmos.

Observando os valores obtidos, torna-se evidente a importância do balanceamento como forma de melhorar o processo produtivo de uma linha de montagem. Analisando primeiramente a melhoria nos indicadores Desbalanceamento e Índice de Suavidade, sendo estas de 74,5 e $67 \%$, respectivamente, verifica-se a eficiência do procedimento de balanceamento em diminuir o tempo ocioso da linha de montagem, através de uma melhor distribuição das tarefas às estações de trabalho. Já o indicador referente à produtividade da linha, obteve uma melhoria de $31 \%$ (menor em relação aos outros indicadores), mas não menos significativa.

Tabela 4. Solução final.

\begin{tabular}{cc}
\hline \multicolumn{2}{c}{ Solução final - segundo passo } \\
\hline Estações de trabalho & Tarefas \\
\hline 1 & $1,2,3,4,6,7$ \\
2 & 5,8 \\
3 & $9,10,11,12,13$ \\
4 & $14,15,17,21$ \\
5 & $16,18,19,22$ \\
6 & $23,24,25,26,27$ \\
7 & $20,28,29,30,31$ \\
8 & $32,33,34,37$ \\
9 & $35,36,38,39,40$ \\
$B_{\mathrm{b}-\mathrm{s}}$ & 0,08 \\
$\mathrm{~B}_{\mathrm{w}-\mathrm{S}}$ & 0,27 \\
\hline
\end{tabular}

\section{Conclusão}

A customização de produtos vem sendo identificada como uma estratégia competitiva promissora por um número crescente de empresas. Associada aos fundamentos da customização em massa, a customização de produtos permite oferecer diversidade aos clientes, por preços similares àqueles atingíveis em cenários de produção massificada. Entretanto, a implementação prática da customização requer a flexibilização do sistema produtivo. Tal flexibilização é particularmente problemática em sistemas organizados em layouts lineares, já que linhas de montagem de produtos costumam ter a sua eficiência prejudicada pela troca constante dos modelos a serem produzidos.

Neste artigo apresentou-se uma metodologia para o balanceamento de linhas de produção multi-modelo. O objetivo principal é proporcionar a flexibilidade requerida pela customização em massa em layouts lineares. A metodologia proposta apresenta duas contribuições relevantes e que não encontram paralelo na literatura. Primeiro, propõe-se uma estrutura de decisão que orienta o usuário quanto à utilização do algoritmo de balanceamento multi-modelo mais adequado a seu cenário de aplicação. Segundo, propõem-se indicadores que permitem quantificar a melhoria resultante do balanceamento da linha multi-modelo. Adicionalmente, apresenta-se uma classificação dos algoritmos de balanceamento multi-modelo na literatura, com base no tratamento dado aos tempos de execução das tarefas que compõem o processo produtivo.

As informações coletadas a respeito da linha de montagem analisada no estudo de caso foram aplicadas ao procedimento desenvolvido por Vilarinho e Simaria (2002) para o balanceamento de linhas de montagem multi-modelo, sendo este escolhido por melhor se adequar à situação em estudo. Como forma de auxiliar na obtenção da solução final, a rotina de cálculos desenvolvida pelos autores foi inserida em uma planilha eletrônica. A fim de comprovar a eficiência do procedimento escolhido, foram utilizados indicadores de desempenho que refletissem as necessidades da linha de montagem analisada. Desse modo, o cálculo dos indicadores utilizados foi realizado antes e após o balanceamento da linha de montagem.

A metodologia mostrou-se eficiente no sentido de otimizar as etapas do processo necessárias à aplicação da ferramenta de balanceamento, bem como na organização e coleta dos dados. Da mesma forma, o algoritmo

Tabela 5. Indicadores de desempenho antes e após o balanceamento.

\begin{tabular}{lccc}
\hline Indicadores & Antes do balanceamento & Após o balanceamento & Melhoria obtida (\%) \\
\hline Produtividade da linha/turno (unid.) & 42 & 55 & 31 \\
Desbalanceamento (\%) & 58,2 & 14,8 & 74,5 \\
Índice de suavidade (min.) & 11,3 & 3,7 & 67 \\
\hline
\end{tabular}


desenvolvido por Vilarinho e Simaria (2002) mostrou-se eficaz na redução do tempo ocioso da linha, bem como no seu aumento de produtividade, sendo que este indicador obteve uma melhora de $31 \%$. A partir da diminuição do tempo ocioso da linha foi possível aumentar sua produção em 13 refrigeradores por turno de trabalho.

A implementação prática do balanceamento na linha de montagem resultou em um baixo custo associado a modificações no sistema produtivo, já que não houve duplicação de postos de trabalho após o balanceamento. O balanceamento reportado neste trabalho foi utilizado pela empresa durante cinco meses, até o momento em que um dos produtos considerados na análise teve sua produção descontinuada. Durante o período analisado, obteve-se a comprovação empírica dos valores apontados para os indicadores na Tabela 5.

O estudo de caso possibilitou uma integração entre os pesquisadores e a empresa onde foi desenvolvido o estudo, proporcionando à mesma benefícios através da metodologia desenvolvida. Desde o início do trabalho a empresa se mostrou interessada, percebendo a possibilidade de otimização do seu sistema produtivo. A resistência dos operadores da linha, inicialmente constatada na etapa de coleta de dados, foi rapidamente superada ao serem expostas as potenciais melhorias obtidas através da implementação do balanceamento.

\title{
Method for balancing mixed model assembly lines in mass customized production systems
}

\begin{abstract}
Product customization requires flexibility from production systems, which is attainable by adopting assembly line configurations able to alternate different models of a same product, i.e., mixed model assembly lines. In these production arrangements, the assignment of tasks for work centers grows in complexity and single model balancing algorithms tend to perform poorly. This article presents a method to balance mixed model assembly lines based on algorithms available in the literature and in original proposals. A classification of balancing algorithms for the mixed model case is also presented, along with their most relevant features. The proposed method is applied to a case study from the home appliances industry.
\end{abstract}

Keywords: Mixed model assembly line balancing. Classification of algorithms. Layout. Optimization.

\section{Referências bibliográficas}

ASKIN, R. G.; ZHOU, M. A parallel station heuristic for the mixed model production line balancing problem. International Journal of Production Research, London (Inglaterra), v. 35, n. 11, p. 3095-3105, 1997.

BECKER, C.; SCHOLL, A. A survey on problems and methods in generalized assembly line balancing. European Journal of Operational Research, London (Inglaterra), v. 168, n. 3, p. 694-715, 2006.

BOCK, S.; ROSENBERG, O.; VAN BRACKEL, T. Controlling mixed-model assembly lines in real-time by using distributed systems. European Journal of Operational Research, London (Inglaterra), v. 168, n. 3, p. 880-904, 2006.

BUKCHIN, J.; DAR-EL, E. M.; RUBINOVITZ, J. Mixed model assembly line design in a make-to-order environment. Computers \& Industrial Engineering, Los Angeles (EUA), v. 41, n. 4, p. 405-421, 2002.

DA SILVEIRA, G.; BORESTEIN, D.; FOGLIATTO, F. S. Mass customization: Literature review and research direction,
International Journal of Production Economics, Linkoping (Suécia), v. 72, n. 1, p. 1-13, 2001.

DAVIS, S. M. "From Future Perfect": Mass Customization. Planning Review, New York (EUA), v. 17, n. 2, p. 16-21, 1989.

EREL, E.; GOKCEN, H. A goal programming approach to mixed-model assembly line balancing problem. International Journal of Production Economics, Linkoping (Suécia), v. 48, n. 2, p. $177-185,1997$.

Shortest-route formulation of mixed-model assembly line balancing problem. European Journal of Operational Research, London (Inglaterra), v. 116, n. 1, p. 194-204, 1999.

EREL, E.; SARIN, S. C. A survey of the assembly line balancing procedures. Production Planning \& Control, Plymouth (Inglaterra), v. 9, n. 5, p. 414-434, 1998.

FRUTOS, J. D.; BORENSTEIN, D. A framework to support customer-company interaction in mass customization environments. Computers in Industry, Groningen (Holanda), v. 54, n. 2 , p. $115-135,2004$. 
GERWIN, D. Manufacturing flexibility: A strategic perspective. Management Science, Evanston (EUA), v. 39, n. 4, p. 395-410, 1993.

GHOSH, S.; GAGNON, R. J. A comprehensive literature review and analysis of the design, balancing and scheduling of assembly systems. International Journal of Production Research, London (Inglaterra), v. 27, n. 4, p. 637-670, 1989.

GUTJAHR, A. L.; NEMHAUSER, G. L. An algorithm for the assembly line balancing problem. Management Science, Evanston (EUA), v. 11, n. 2, p. 308-315, 1964.

HAQ, A. N.; JAYAPRAKASH, J.; RENGARAJAN, K. A hybrid genetic algorithm approach to mixed-model assembly line balancing. International Journal of Advanced Manufacturing Technology, v. 28, n. 3-4, p. 337-341, 2005.

JIAO, J.; MA, Q.; TSENG, M. M. Towards high value-added products and services: mass customization and beyond. Technovation, Ottawa (Canadá), v. 23, n. 10, p. 809-821, 2003.

KARABATI, S.; SAYIN, S. Assembly line balancing in a mixed model sequencing environment with synchronous transfer. European Journal of Operational Research, London (Inglaterra), v. 149, n. 2, p. 417-429, 2003.

KHAN, A.; DAY, A. J. A Knowledge Based Design Methodology for manufacturing assembly lines. Computers \& Industrial Engineering, Los Angeles (EUA), v. 41, n. 4, p. 441-467, 2002.

KILBRIDGE, M. D.; WESTER, L. A heuristic method of assembly line balancing. Journal of Industrial Engineering, Cincinnatti (EUA), v. 12, n. 4, p. 292-298, 1961.

KIM, Y. K., KIM, S. J.; KIM, J. Y. Balancing and sequencing mixed model U-lines with a co-evolutionary algorithm. Production Planning \& Control, Plymouth (Inglaterra), v. 11, n. 8, p. 754-764, 2000a.

.A Coevolutionary Algorithm for Balancing and Sequencing in Mixed Model Assembly Lines. Applied Intelligence, Houston (EUA), v. 13, n. 3, p. 247-258, 2000b.

An endosymbiotic evolutionary algorithm for the integration of balancing and sequencing in mixed-model Ulines. European Journal of Operational Research, London (Inglaterra), v. 168, n. 3, p. 838-852, 2006.

LAMPEL, J.; MINTZBERG, H. Customizing customization. Sloan Management Review, Cambridge (EUA), v. 38, n. 1, p. 21-30, 1996.

LEHMAN, M. On criteria for assigning models to assembly lines. International Journal of Production Research, London (Inglaterra), v. 7, n. 4, p. 269-285, 1969.

MACASKILL, J. L. C. Production line balances for mixed model lines. Management Science, Evanston (EUA), v. 19, n. 4, p. 423-434, 1972.
MATANACHAI, S.; YANO, C. A. Balancing mixed model assembly line to reduce work overload. IIE Transactions, Berkeley (EUA), v. 33, n. 1, p. 29-42, 2001.

McMULLEN, P. R.; FRAZIER, G. V. A heuristic for solving mixedmodel line balancing problems with stochastic task duration and parallel stations. International Journal of Production Economics, Linkoping (Suécia), v. 51, n. 3, p. 177-190, 1997.

McMULLEN, P. R.; TARASEWICH, P. Using ant techniques to solve the assembly line balancing problem. IIE Transactions, Berkeley (EUA), v. 35, n. 7, p. 605-617, 2003.

MERENGO, C.; NAVA, F.; POZZETTI, A. Balancing and sequencing manual mixed-model assembly lines. International Journal of Production Research, London (Inglaterra), v. 37, n. 12, p. 2835-2860, 1999.

MILTENBURG, J. Balancing and Scheduling Mixed-Model UShaped Production Lines. International Journal of Flexible Manufacturing Systems, Richardson (EUA), v. 14, n. 2, p. 119-151, 2002.

PASTOR, R. et al. Tabu search algorithms for an industrial multi-product and multi-objective assembly line balancing problem, with reduction of the task dispersion. Journal of the Operational Research Society, Birmingham (Inglaterra), v. 53, n. 12, p. 1317-1323, 2002.

SARKER, B. R.; PAN, H. Designing a mix-model assembly line to minimize the costs of idle and utility times. Computers \& Industrial Engineering, Los Angeles (EUA), v. 34, n. 3, p. 609-628, 1998.

SPARLING, D.; MILTENBURG, J. The mixed model U-line balancing problem. International Journal of Production Research, London (Inglaterra), v. 36, n. 2, p. 485-501, 1998.

SPRECHER, A. Dynamic search tree decomposition for balancing assembly lines by parallel search. International Journal of Production Research, London (Inglaterra), v. 41, n. 7, p. 1413-1430, 2003.

THOMOPOULOS, N. T. Line balancing-sequencing for mixed model assembly. Management Science, Evanston (EUA), v. 14, n. 2, p. B59-B75, 1967.

Mixed model line balancing with smoothed station assignment. Management Science, Evanston (EUA), v. 16, n. 9, p. 593-603, 1970.

VAN ZANTE-DE FOKKERT, J. I.; DE KOK, T. G. The mixed and multi model line balancing problem: a comparison. European Journal of Operational Research, London (Inglaterra), v. 100, n. 3, p. 399-412, 1997.

VILARINHO, P.; SIMARIA, A. S. A two-stage heuristic method for balancing mixed-model assembly lines with parallel workstations. International Journal of Production Research, London (Inglaterra), v. 40, n. 6, p. 1405-1420, 2002. 


\section{Sobre os autores}

\section{Melissa Petry Gerhardt}

\section{Flávio Sanson Fogliatto}

Laboratório de Otimização de Produtos e Processos - LOPP,

Departamento de Engenharia de Produção e Transportes, Escola de Engenharia,

Universidade Federal do Rio Grande Do Sul - UFRGS,

Av. Osvaldo Aranha, 99, $5^{\circ}$ Andar, Porto Alegre, Rs, Brasil,

e-mails: melissa@ producao.ufrgs.br; ffogliatto@producao.ufrgs.br

\section{Marcelo Nogueira Cortimiglia}

Programma di Dottorato di Ricerca, Dipartimento di Ingegneria Gestionale, Politecnico Di Milano,

Via Giuseppe Colombo, 40 20133, Milão, Itália,

e-mail: marcelo.cortimiglia@mail.polimi.it

Agradecimentos: Ao Cnpq Pelo Apoio Ao Prof. Fogliatto (Grant \#301433/2005-4).

Recebido em 22/12/2005

Aceito em 11/1/2007 
